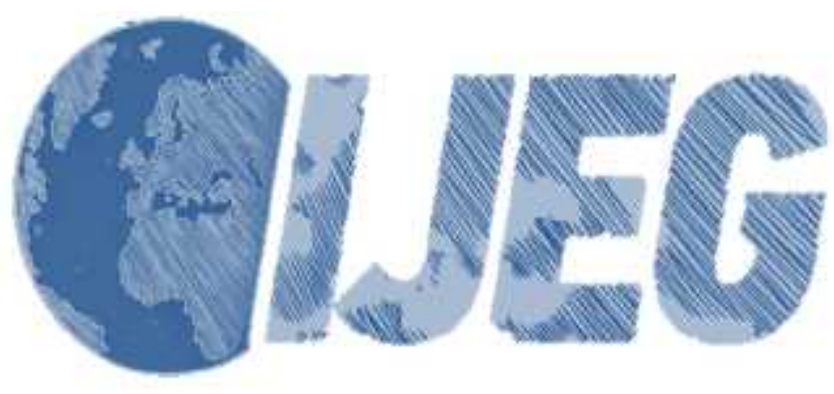

International Journal of Engineering and Geosciences (IJEG),

Vol; 2; , Issue; 03, pp. 91-99, October, 2017, ISSN 2548-0960, Turkey,

DOI: $10.26833 /$ ijeg. 321278

\title{
VALUATION OF RESIDENCES THROUGH UTILIZING RATIO OF INTEGRATED CAPITALISATION
}

\author{
Ertas, M., ${ }^{1 *}$ Bayındir, B., ${ }^{2}$ \\ ${ }^{1}$ Selçuk University, Technical Sciences Vocational Higher School, City and Region Planning Department, Map \& \\ Cadastre Program, Konya, Turkey (mertas@ selcuk.edu.tr); \\ ${ }^{2}$ Energy \& Natural Resources Ministry, Türkiye Electricity Distribution Inc., Aras Region Directorat, Erzurum, Turkey \\ (bahadirbayindir24@hotmail.com);
}

ID ORCID: 0000-0002-9458-3395; ID ORCID: 0000-0003-4033-346X

*Corresponding Author, Received: 13/06/2017, Accepted: 03/08/2017

\begin{abstract}
Right after the Second World War, determining the value of real properties gained importance in the countries, in which citizens had the right for real property possession. As for our country, importance of evaluating real estates noticed in late 1990's. Now in Turkey, evaluation is the only method for the determining of the market value of real properties used by private and public sector.

Today, several methods have been used for evaluating real properties. One of them is the ratio of integrated capitalisation within income method. This method provides quite fast and accurate results for bankers, real estate consultants and judicial system operating in related market.

Through using this method, evaluation of the residences in a region, located in Centre District, Province of Erzincan is aimed. Selling and renting values have been researched for integrated capitalisation ratios in evaluation of residences located in project field. Through utilizing integrated capitalisation ratios; market values of the residences, located in project field and whose revenues are known, can be determined. In the same way, through utilizing related ratio, current rental values of residences located in project field and whose selling values are known can be determined.
\end{abstract}

Keywords: Valuation, Integrated Capitalisation, Income Method, Capitalisation Rate 


\section{INTRODUCTION}

Nowadays, real properties are also regarded as an investment tool along with human's basic needs like shelter and trade. Being valuable, originated from ownership and ownership originated rights of real properties used for living and trade purposes, requires a need for determination of real properties' possible trade values.

Properties which are not possible to move one place to another and fixed in current location like land, building site and building (house/flat/residence, apartment, factory/plant/facility etc.) are called real properties/estates.

As for evaluation of real properties; it means to determine the value of a real property in a definite time objectively, independently and neutrally by evaluating agents related to real property like characteristic, utility, neighbourhood, using conditions.

It comprises independent and neutral evaluation of possible value of a real property, a real property project or rights and benefits originating from a real property in a definite time (UDES).

It is the prediction of defined value in evaluation date of a real property, a real property project or real rights based on independent, neutral and unbiased criteria (Çağdaş, 2012).

According to Capital Market Committee, evaluation; "is to independently and neutrally determine possible value of a real property, a real property project or rights and benefits originating from a real property in a definite time.

\section{METHODS FOR VALUATION OF REAL PROPERTY}

Real properties can be evaluated one of three widely used evaluation methods. These three main methods have also sub-groups. These sub-groups are;

a) Comparison,

b) Income,

c) Cost

Purpose of the comparison methods to evaluate related real property through analysis of comparable sample real properties. Urban area and development data are basic comparison criteria. Fort this reason, samples and building sites whose values will be determined should be in the same region and possess same characteristic and similar development data.

To sum up, It means to determine the current value by comparing realised sales outcomes. Method can be especially applied for unconstructed parcels or partly constructed parcels. It requires getting generally attainable information from land registers, sale contracts, building contracts in which flat(s) or floor(s) is given in return to landowner, Chamber of Real Estate Agents' Lowest Price Lists, Capital Market Committee Registrations, National Property sales and loan agreements (Ertaş 2015).

Parcels should be appropriate with each other compared to location, quality, density and price levels. So, basically, according to similarities of fundamental data, a price comparison is realised. Considering the evaluations mentioned above, features of evaluating and comparing parcels are written down suggested norm table.The point not to be forgotten is that, parcel qualities should indicate adequate similarities with each other.

Basis of income method in a constructed parcel is the net income that can be acquired continuously from land and its building facilities. Because of limited using period of a building and unlimited using period of land; while turning net revenue into money, its equivalent to

a) Ground,

b) Building and

c) Additional facilities

is separated. Principally, value of ground is determined by "comparison method" (Köktürk ve ark).

Income Method is appropriate for evaluation of constructed parcels and rental revenue getting buildings like apartments, stores and office blocks by preference. Income isn't equal to the gross rental value's money equivalent. Income Method is also quite sensitive in letting predictions. Expert should be experienced to be able to decide usual rental value in a neighbourhood by utilizing related method.

In income value determining;

a) Construction year and economic using period,

b) Gross and net amount of revenue,

c) Square meter prices of each floors,

d) Sum of residential/housing areas

should be carefully designated.

As for Cost Method, it's appropriate for detached or semi-detached buildings/houses, industrial facilities and public buildings.

Object $($ cost $)$ value $=$ ground value + building (production along with additional facilities and business properties) value- technical deterioration (originating from functional or economic deterioration) value losses.

Production cost, by using building indexes, can be converted into evaluation date values. Value losses sourced by aging depend on remaining using period of building facilities. Building object value, production expenses and remaining economic using period are crucial. Ground value is depended on construction permission given according to development plan and related act \& regulations. It is also depended on regional location, traffic condition, infrastructure expenditures and using opportunities.

\section{COMPARISON METHOD WITH RATIO OF INTEGRATED CAPITALISATION}

Capitalisation Ratio is defined as the ratio that turns real properties annual net operating income into market value. As for integrated capitalisation ratio, it's determined through dividing highly similar real properties' (land + building + equipment's) net income by sale price in local market (Ertaş, 2016).

Integrated capitalisation is calculated through dividing highly similar real properties' net incomes by sale values in local market (1). 
$k_{\text {bït }}=\left(\frac{\frac{G_{1}}{D_{1}}+\frac{G_{2}}{D_{2}}+\ldots+\frac{G_{n}}{D_{n}}}{n}\right)$

correlation is taken into consideration. When this correlation is divided into its compounds (land + building + equipment's), correlation no. (2) is obtained.

$$
k_{\text {biut }}=k_{a} \frac{D_{a}}{D_{t a s}}+k_{y} \frac{D_{y}}{D_{t a s}}=\frac{G_{\text {tnet }}}{D_{\text {tas }}}
$$

Comparison Method by utilizing integrated capitalisation ratio is applied by using comparable real properties' sale and net income values that are similar compared to building type and its age, type of residence, using type, its location, etc. Firstly, real properties are classified according to various qualities. Applying for each class separately, classified real properties' net income and sale values are examined. By using obtained data, real properties' capitalisation ratios are calculated and their means are solved. So, mean ratio for the related class is determined. This ratio provides us to evaluate similar real properties without dividing them into their compounds.

Net income is calculated by 3 correlations.

$$
D_{\text {knet }}=D_{k y l}-Z G_{k i r a}
$$

\section{APPLICATIONS}

By utilizing determined integrated capitalisation ratio of similar residences located in Project field (Map 1) composed of a part of Yavuz Selim Neighbourhood, Province of Erzincan, Centre District, and small part of Demirkent Municipality; evaluation of other real properties in the same region is aimed.

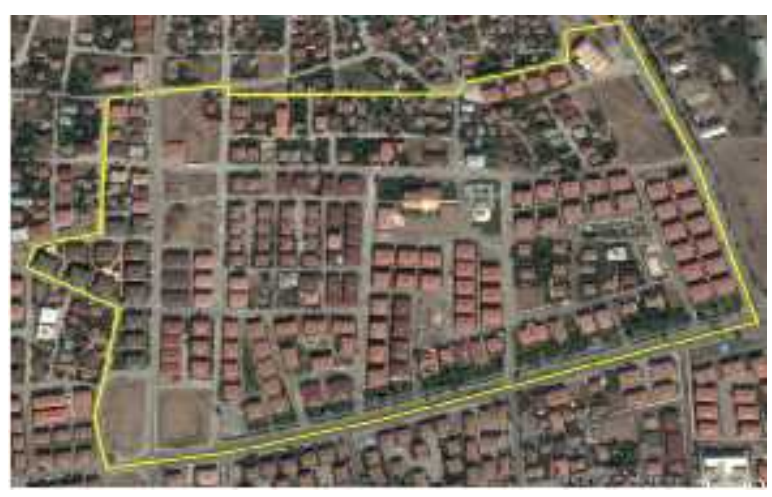

Map 1 Project Field

Determining market value of real properties are required for a great many of transactions like loan, mortgage, tax, trade, renting, nationalization. So, fair evaluation of real properties to preserve the rights of related parties has a great importance. In evaluation process, preferred method and considered objective values are formed the first procedure steps to determine real value of properties. In practice, reason for preference of comparison method with integrated capitalisation ratio is that almost all residences located in project field are highly similar buildings with each other.

This method cannot be used when there is no measurable data available.

\section{Gathering Data}

Essential data for evaluation are gathered by utilizing survey table shown in Table 1 . Renting value, sale value / time, age, data shown under the title of isolation, real properties' owners, inhabitants shown in prepared table are obtained by statements of local real estate agents.

Table 1 Survey Table (including information of real

\begin{tabular}{|c|c|c|c|}
\hline \multicolumn{3}{|l|}{ Flat } & Building No: \\
\hline $\operatorname{Area}\left(\mathbf{m}^{2}\right)$ & $\begin{array}{c}\text { Rental } \\
\text { Value(TL) }\end{array}$ & $\begin{array}{c}\text { Sale } \\
\text { Value/Year }\end{array}$ & $\frac{\text { Construction }}{\text { Type }}$ \\
\hline Age & & & $\begin{array}{ll}\text { Masonry } \\
\text { Wooden } \\
\text { Prefabricated }\end{array}$ \\
\hline Floor No & Isolation & Frontage & Residence Type \\
\hline $\begin{array}{ll}\text { Ground } & \square \\
\text { Top } & \\
\text { Intermediate } & \square\end{array}$ & $\begin{array}{l}\text { Present } \square \\
\text { Absent } \square\end{array}$ & $\begin{array}{ll}\text { East } & \square \\
\text { West } & \square \\
\text { North } & \square \\
\text { South } & \square\end{array}$ & $\begin{array}{l}\text { Detached } \\
\text { Housing Estate } \\
\text { Apartment }\end{array}$ \\
\hline Numberof Floor & $\frac{\text { Road Width }}{\underline{(\mathrm{m})}}$ & \begin{tabular}{l}
\multicolumn{2}{|c}{ Parking/ } \\
Garage \\
Present $\square$ \\
Absent $\square$
\end{tabular} & $\underline{\operatorname{Garden}\left(\mathrm{m}^{2}\right)}$ \\
\hline $\begin{array}{c}\text { Closest } \\
\text { Attraction Type }\end{array}$ & $\begin{array}{l}\frac{\text { Distance to }}{\underline{\text { Closest }}} \\
\frac{\text { Attraction }}{\underline{(m)}}\end{array}$ & $\begin{array}{l}\frac{\text { Location in }}{\text { Block }} \\
\text { Corner } \\
\text { Intermediate }\end{array}$ & \begin{tabular}{|l}
\multicolumn{2}{|c}{ Landscape } \\
Present $\square$ \\
Absent $\square$
\end{tabular} \\
\hline hers & & & \\
\hline
\end{tabular}
property)

Development plans obtained from related municipalities is evaluated in NETCAD program and data titles called as area, road width, garden, distance to closest attraction point are achieved shown in table. Data called as "Other" and shown under the related title in table is achieved from physical researchs in project field.

Over 150 data have been collected in the project area nevertheless data exceeding $10 \%$ of the average value are eliminated and only 120 data were used.

A separate table is prepared for every flat, located in project field, which forms data for evaluation study. Buildings located in project field and their flats are numbered according to cadastre parcel number principles after connecting with municipality development plans (Map 2). 


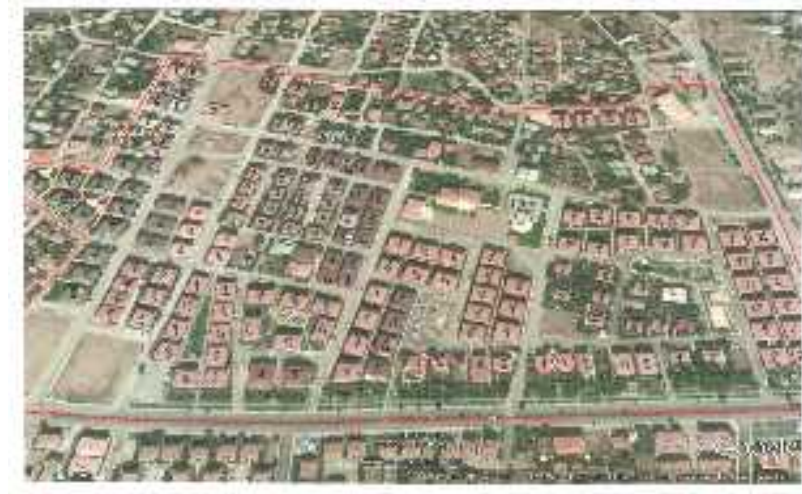

Map 2 Residences in Project Field (in numbered condition)

\subsection{Calculating Data}

Similar real properties located in Project Field, firstly regarding their location (intermediate parcel and corner parcel), then regarding their floor (ground floor, intermediate floor, top floor), are classified. Integrated capitalisation ratio of each class is calculated separately.
While calculating capitalisation ratio of similar real properties located in Project Field; last five year's selling values and current rental values of real properties are used. Sales in 2016 are accepted as sales in April, sales before 2016 are accepted as sales in June of related years. Consumer Price Index (TÜFE) is applied for updating sale prices related to the sales realised before 2016.For the reason that urbanization and planning processes have not been completed yet in our country, real property value increases more than TÜFE Index. For this reason, TÜFE index of 2016 is taken into consideration and its weight is reduced throughout past years. Last five years are studied to diminish possible faults. In updating calculation, correlation no. 4 is used in coefficient of weight.

$$
p=\frac{1}{2016-y l l} \quad D_{s g d}=D_{s} \cdot p
$$

Table 2 Ground Floor - Corner Apartments

\begin{tabular}{|c|c|c|c|c|c|c|c|c|c|c|c|c|c|c|c|}
\hline 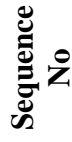 & 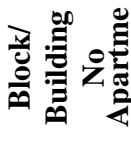 & & $\begin{array}{c}\mathbf{F} \\
\left(\mathbf{m}^{2}\right)\end{array}$ & $D_{\text {kyll }}$ & D knet $_{\text {t }}$ & D $_{\text {kbr }}$ & $\mathbf{D}_{\mathrm{s}}$ & $D_{\text {syll }}$ & $D_{\text {sgd }}$ & $D_{\text {snet }}$ & D $_{\text {sbr }}$ & k (\%) & CY & $\mathbf{A K}$ & $\begin{array}{l}\mathbf{U}_{\mathbf{c}} \\
(\mathbf{m})\end{array}$ \\
\hline $\mathrm{S}, 1$ & $2190 / 4$ & 2 & 152 & 9900 & 9306 & 61,22 & 155000 & 2013 & 193213,53 & 179688,58 & 1182,16 & 5,179 & G - D & Kş. & $S M-260$ \\
\hline $\mathrm{S}, 2$ & $2146 / 5$ & 2 & 175 & 9900 & 9306 & 53,18 & 170000 & 2014 & 194123,19 & 180534,56 & 1031,63 & 5,155 & G - D & Kş. & $\mathrm{ÇOP}-20$ \\
\hline$S, 6$ & $2147 / 1$ & 1 & 155 & 8880 & 8347,2 & 53,85 & 158000 & 2014 & 180420,37 & 167790,95 & 1082,52 & 4,976 & B - K & Kş. & ÇOP - 90 \\
\hline $\mathrm{S}, 8$ & $2087 / 1$ & 2 & 162 & 9000 & 8460 & 52,22 & 163000 & 2014 & 186129,88 & 173100,79 & 1068,52 & 4,887 & $\mathrm{~K}-\mathrm{B}$ & Kş. & $\mathrm{O}-270$ \\
\hline $\mathrm{S}, 8$ & $2087 / 4$ & 1 & 162 & 8760 & 8234,4 & 50,83 & 146000 & 2013 & 181994,68 & 169225,05 & 1044,78 & 4,865 & D - G & Kşs. & $O-200$ \\
\hline$S, 10$ & $2085 / 1$ & 1 & 156 & 8400 & 7896 & 50,62 & 175000 & 2016 & 175000,00 & 162750,00 & 1043,27 & 4,852 & B - K & Kşs. & $\mathrm{O}-80$ \\
\hline $\mathrm{S}, 11$ & $2015 / 5$ & 1 & 167 & 11400 & 10716 & 67,17 & 206000 & 2014 & 235231,63 & 218765,41 & 1309,97 & 4,898 & $D-G$ & Kş. & PYA - 10 \\
\hline $\mathrm{S}, 12$ & $2022 / 10$ & 1 & 149 & 8400 & 7896 & 52,99 & 145000 & 2014 & 165575,66 & 153985,36 & 1033,46 & 5,128 & $G-B$ & Kşs. & $\mathrm{C}-30$ \\
\hline$S, 14$ & $\mathrm{D}, 106 / 3$ & 1 & 160 & 9600 & 9024 & 56,4 & 166000 & 2014 & 189555,58 & 176286,69 & 1101,79 & 5,119 & $G-B$ & Kş. & $\mathrm{SM}-225$ \\
\hline$S, 15$ & $1858 / 5$ & 1 & 131 & 9000 & 8460 & 64,58 & 171000 & 2015 & 182142,58 & 169392,60 & 1293,07 & 4,994 & G - B & Kş. & PYA - 5 \\
\hline$S, 16$ & $1584 / 1$ & 2 & 100 & 4800 & 4512 & 45,12 & 72000 & 2014 & 82216,88 & 76461,70 & 764,62 & 5,901 & D - K & Kşs. & PYA - 75 \\
\hline$S, 16$ & $1586 / 5$ & 1 & 100 & 4680 & 4399,2 & 43,99 & 73000 & 2014 & 83358,78 & 77523,67 & 775,24 & 5,675 & $K-D$ & Kş. & PYA - 70 \\
\hline $\mathrm{S}, 17$ & $2190 / 1$ & 3 & 153 & 9300 & 8742 & 57,14 & 195000 & 2016 & 195000 & 181350 & 1185,29 & 4,821 & K - B & Kş. & PYA - 10 \\
\hline $\mathrm{S}, 17$ & $2190 / 5$ & 1 & 153 & 9600 & 9024 & 58,98 & 190000 & 2015 & 202380,64 & 188214,00 & 1230,16 & 4,795 & G - B & Kş. & $S M-200$ \\
\hline $\mathrm{A}, 4$ & $2088 / 1$ & 1 & 100 & 8400 & 7896 & 78,96 & 175000 & 2016 & 175000 & 162750 & 1627,50 & 4,852 & G - B & Kş. & PYA - 3 \\
\hline AVR. & & & 145 & 8668 & 8148 & 56,28 & 157333,3 & & 174756,23 & 162521,29 & 1118,27 & 5,073 & & & \\
\hline
\end{tabular}


Table 3 Ground Floor - Intermediate Apartments

\begin{tabular}{|c|c|c|c|c|c|c|c|c|c|c|c|c|c|c|c|}
\hline 氖 & 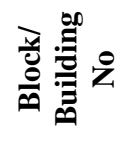 & 总 & $\stackrel{\ominus}{\mathbf{Z}}_{\left(\mathbf{m}^{2}\right)}^{\mathbf{F}}$ & $D_{\text {kyl }}$ & D knet & $D_{\text {kbr }}$ & $\mathbf{D}_{\mathrm{s}}$ & $D_{\text {syl }}$ & $\mathbf{D}_{\text {sgd }}$ & $D_{\text {snet }}$ & $\mathbf{D}_{\text {sbr }}$ & k $(\%)$ & CY & AK & $\begin{array}{l}\mathbf{U}_{\mathbf{c}} \\
(\mathbf{m})\end{array}$ \\
\hline $\mathrm{S}, 1$ & $1591 / 1$ & 1 & 152 & 9000 & 8460 & 55,66 & 170000 & 2015 & 181077,42 & 168402 & 1 107,91 & 5,024 & $K-D$ & Ar. & $S M-245$ \\
\hline $\mathrm{S}, 1$ & $1591 / 3$ & 2 & 152 & 9600 & 9024 & 59,37 & 178000 & 2015 & 189598,71 & 176326,81 & 1160,04 & 5,118 & G - D & Ar. & $S M-270$ \\
\hline $\mathrm{S}, 1$ & $2190 / 2$ & 1 & 152 & 9300 & 8742 & 57,51 & 149000 & 2013 & 185734,30 & 172732,89 & 1136,40 & 5,061 & G - B & Ar. & $S M-210$ \\
\hline $\mathrm{S}, 1$ & $2190 / 3$ & 1 & 152 & 9300 & 8742 & 57,51 & 148000 & 2013 & 184487,76 & 171573,61 & 1128,77 & 5,095 & $G-B$ & Ar. & $\mathrm{SM}-235$ \\
\hline $\mathrm{S}, 2$ & $2146 / 7$ & 2 & 175 & 9000 & 8460 & 48,34 & 171000 & 2015 & 182142,58 & 169392,60 & 967,96 & 4,994 & G - D & Ar. & ÇOP - 30 \\
\hline $\mathrm{S}, 4$ & $\mathrm{D}, 1840 / 1$ & 1 & 161 & 8400 & 7896 & 49,04 & 150000 & 2014 & 171285,17 & 159295,20 & 989,41 & 4,957 & K - D & Ar. & $C-250$ \\
\hline $\mathrm{S}, 4$ & $\mathrm{D}, 1840 / 3$ & 1 & 161 & 8520 & 8008,8 & 49,74 & 140000 & 2013 & 174515,45 & 162299,36 & 1008,07 & 4,935 & K - D & Ar. & $C-280$ \\
\hline $\mathrm{S}, 5$ & $1858 / 2$ & 2 & 172 & 7200 & 6768 & 39,35 & 100000 & 2011 & 146969,37 & 136681,52 & 794,66 & 4,952 & $G-D$ & Ar. & $S M-300$ \\
\hline $\mathrm{S}, 5$ & $1858 / 3$ & 1 & 172 & 7200 & 6768 & 39,35 & 115000 & 2013 & 143351,97 & 133317,33 & 775,10 & 5,077 & G - B & Ar. & $\mathrm{SM}-320$ \\
\hline $\mathrm{S}, 5$ & $2016 / 1$ & 1 & 172 & 6960 & 6542,4 & 38,04 & 104000 & 2012 & 140396,95 & 13056 & 759,12 & 5,011 & $D-G$ & Ar. & SM - 290 \\
\hline $\mathrm{S}, 5$ & $2016 / 4$ & 2 & 172 & 7080 & 6655,2 & 38,69 & 125000 & 2014 & 142737,64 & 132746,00 & 771,78 & 5,013 & B - G & Ar. & $\mathrm{O}-340$ \\
\hline $\mathrm{S}, 5$ & $2016 / 5$ & 2 & 172 & 7200 & & 39,35 & 110000 & 2012 & 148496,78 & 13810 & & 901 & B - G & Ar. & $\mathrm{O}-350$ \\
\hline $\mathrm{S}, 5$ & $2016 / 9$ & 2 & 172 & 6900 & 6486 & 37,71 & 110000 & 2013 & 137119,28 & 127520,93 & 741,40 & 5,086 & D - K & Ar. & $\mathrm{SM}-350$ \\
\hline $\mathrm{S}, 5$ & $2016 / 10$ & 1 & 172 & 6960 & 6542,4 & 38,04 & 115000 & 2013 & 143351,97 & 133317,33 & 775,10 & 4,907 & $D-G$ & Ar. & $\mathrm{SM}-340$ \\
\hline S,6 & $2147 / 7$ & 1 & 155 & 8400 & & 50,94 & 120000 & 2011 & 176363,25 & 16401 & 105 & & B - K & Ar. & ÇOP -150 \\
\hline $\mathrm{S}, 6$ & $2147 / 8$ & 2 & 155 & 8700 & 8178 & 52,76 & 145000 & 2013 & 180748,14 & 168095,77 & 1084,49 & 4,865 & B - G & Ar. & $\mathrm{ÇOP}-120$ \\
\hline $\mathrm{S}, 8$ & $2087 / 2$ & 1 & 162 & 8700 & 8178 & 50,48 & 135000 & 2012 & 182246,04 & 169488,82 & 1046,23 & 4,825 & K - D & Ar. & $\mathrm{O}-240$ \\
\hline $\mathrm{S}, 8$ & $2087 / 6$ & 1 & 162 & 8400 & & 48,74 & 1400 & 2013 & 1745 & 162299,36 & 1001,85 & 5,074 & B - K & Ar. & $O-280$ \\
\hline $\mathrm{S}, 9$ & $2021 / 1$ & 2 & 200 & 9600 & 9024 & 45,12 & 162000 & 2013 & 201939,30 & 187803,55 & 939,02 & 4,805 & G - D & Ar. & $C-230$ \\
\hline $\mathrm{S}, 9$ & $2021 / 3$ & 2 & 200 & 9900 & 9306 & 46,53 & 155000 & 2012 & 209245,46 & 194598,28 & 972,99 & 4,782 & $G-D$ & Ar. & $C-300$ \\
\hline $\mathrm{S}, 9$ & $2021 / 8$ & 2 & 200 & 10560 & 9926,4 & 49,63 & 194000 & 2014 & 221528,81 & 206021,80 & 1030,11 & & $G-D$ & Ar. & PYA - 10 \\
\hline $\mathrm{S}, 9$ & $2022 / 5$ & 1 & 200 & 9600 & 9024 & 45,12 & 166000 & 2013 & 206925,46 & 192440,67 & 962,20 & 4,690 & $G-B$ & Ar. & $\mathrm{C}-180$ \\
\hline $\mathrm{S}, 11$ & $2015 / 4$ & 2 & 167 & 10800 & 10152 & 60,79 & 185000 & 2013 & 230609,70 & 214467,02 & 1284,23 & 4,734 & B - G & Ar. & PYA - 10 \\
\hline $\mathrm{S}, 12$ & $2022 / 10$ & 2 & 149 & 8160 & 7670,4 & & & 2013 & & 147228,71 & & 5,210 & G - B & Ar. & $\mathrm{C}-30$ \\
\hline $\mathrm{S}, 13$ & $2086 / 1$ & 2 & 100 & 6300 & 5922 & 59,22 & 111000 & 2014 & 126751,02 & 117878,45 & 1178,78 & 5,024 & $G-D$ & Ar. & $O-130$ \\
\hline $\mathrm{S}, 13$ & $2086 / 3$ & 1 & 100 & 6600 & 6204 & 62,04 & 117000 & 2014 & 133602,43 & 124250,26 & 1242,50 & 4,993 & G - B & Ar. & $\mathrm{O}-130$ \\
\hline $\mathrm{S}, 14$ & $\mathrm{D}, 105 / 4$ & 1 & 160 & 9000 & & & & 2015 & 175751,61 & 163449,00 & 1021,56 & 5,176 & G - B & Ar. & $S M-120$ \\
\hline $\mathrm{S}, 14$ & $\mathrm{D}, 105 / 7$ & 2 & 160 & 8820 & 8290,8 & 51,82 & 150000 & 2014 & 171285,17 & 159295,20 & 995,60 & 5,205 & $G-D$ & Ar. & $\mathrm{SM}-130$ \\
\hline $\mathrm{S}, 15$ & $1858 / 7$ & 1 & 131 & 7800 & 7332 & 55,97 & 153000 & 2015 & 162969,67 & 151561,80 & 1156,96 & 4,838 & G - B & Ar. & $S M-260$ \\
\hline$S, 16$ & $1586 / 8$ & 1 & 100 & 4620 & 4342,8 & 43,43 & & 2015 & & & & 5,694 & $D-G$ & Ar. & PYA - 20 \\
\hline$A, 6$ & $1584 / 7$ & 1 & 114 & 6600 & 6204 & 54,42 & 110000 & 2013 & 137119,28 & 127520,93 & 1118,60 & 4,865 & G - B & Ar. & $\mathrm{O}-25$ \\
\hline A, 6 & $2085 / 6$ & 2 & 112 & 7200 & 6768 & 60,43 & & 2015 & 143796,77 & 133731,00 & 1 194,03 & 5,051 & $B-G$ & Ar. & $O-110$ \\
\hline $\mathrm{A}, 7$ & $1584 / 3$ & 1 & 132 & 7800 & 7332 & 55,55 & 160000 & 2016 & 160000 & 148800 & 1127,27 & 4,927 & $B-K$ & Ar. & $\mathrm{O}-150$ \\
\hline $\mathrm{A}, 8$ & $1858 / 1$ & 1 & 162 & 10800 & 10152 & 62,67 & 187000 & 2014 & 213535,51 & 198588,02 & 1225,85 & 5,112 & K - D & Ar. & $S M-250$ \\
\hline AVR. & & & 156,88 & 8264,12 & 7768,27 & 50,23 & 140559 & & 168250,77 & 156473,22 & 23420,86 & 4,989 & & & \\
\hline
\end{tabular}


Table 4 Intermediate Floor(s) - Corner Apartments

\begin{tabular}{|c|c|c|c|c|c|c|c|c|c|c|c|c|c|c|c|}
\hline 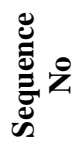 & 궐류 & 竞 & $\underset{\left(\mathbf{m}^{2}\right)}{\mathbf{F}}$ & $D_{\text {kyl }}$ & $D_{\text {knet }}$ & $D_{\text {kbr }}$ & $\mathbf{D}_{\mathrm{s}}$ & $D_{\text {syll }}$ & $D_{\text {sgd }}$ & $D_{\text {snet }}$ & $\mathbf{D}_{\text {sbr }}$ & k $(\%)$ & CY & AK & $\begin{array}{l}\mathbf{U}_{\mathbf{c}} \\
(\mathbf{m})\end{array}$ \\
\hline $\mathrm{S}, 1$ & $2190 / 4$ & 4 & 152 & 10440 & 9813,6 & 64,56 & 168000 & 2013 & 209418,53 & & 1281,31 & 5,039 & G - D & Kș. & \\
\hline $\mathrm{S}, 2$ & $2146 / 1$ & 3 & 175 & 9600 & 9024 & 51,57 & 186000 & 2015 & 198119,99 & 184251,60 & 1052,87 & 4,898 & $G-B$ & Kş. & ÇOP -10 \\
\hline $\mathrm{S}, 3$ & $2145 / 1$ & 3 & 181 & 10200 & 9588 & 52,97 & 172000 & 2013 & 214404,69 & 199396 & 1101,64 & 4,808 & G - B & Kş. & $\mathrm{O}-60$ \\
\hline S,5 & $2016 / 7$ & 3 & 172 & 9000 & 8460 & 49,19 & 145000 & 2013 & 180748,14 & 168095,77 & 977,30 & 5,033 & G - B & Kş. & PYA - 3 \\
\hline$S, 6$ & $2145 / 6$ & 3 & 155 & 9600 & 9024 & 58,22 & 183000 & 2015 & 194924,51 & 181279,80 & 1169,55 & 4,978 & $G-B$ & Kş. & ÇOP - 55 \\
\hline $\mathrm{S}, 6$ & $2147 / 1$ & 3 & 155 & 9420 & 8854,8 & 57,13 & 171000 & 2014 & 195265,09 & 181596,53 & 1171,59 & 4,876 & $B-K$ & Kş. & ÇOP - 90 \\
\hline $\mathrm{S}, 7$ & $2147 / 5$ & 4 & 188 & 12000 & 11280 & 60 & 200000 & 2014 & 228380,22 & 212393,60 & 1129,75 & 5,311 & B $-\mathrm{K}$ & Kş. & PYA - 5 \\
\hline $\mathrm{S}, 8$ & $2087 / 4$ & 3 & 162 & 9900 & 9306 & 57,44 & 185000 & 2015 & 202380,64 & 188214,00 & 1161,81 & 4,944 & $D-G$ & $\mathrm{Kşs.}$ & $\mathrm{O}-200$ \\
\hline $\mathrm{S}, 9$ & $2021 / 7$ & 4 & 200 & 10800 & 10152 & 50,76 & 201000 & 2015 & 214097,41 & 199110,59 & 995,55 & 5,099 & G - D & Kş. & PYA - 10 \\
\hline $\mathrm{S}, 10$ & $2085 / 1$ & 3 & 156 & 9600 & 9024 & 57,85 & 169000 & 2014 & 192981,29 & 179472,60 & 1150,46 & 5,028 & B $-\mathrm{K}$ & Kş. & $\mathrm{O}-80$ \\
\hline$S, 13$ & $2086 / 1$ & 3 & 124 & 7800 & 7332 & 59,13 & 153000 & 2015 & 162969,67 & 151561,80 & 1222,27 & 4,838 & $G-B$ & $\mathrm{Kşs.}$ & ÇOP - 115 \\
\hline $\mathrm{S}, 14$ & $\mathrm{D}, 106 / 3$ & 4 & 160 & 10200 & 9588 & 59,92 & 209000 & 2016 & 209000 & 194370 & 1214,81 & 4,933 & G - D & Kș. & $\mathrm{SM}-250$ \\
\hline$S, 15$ & $1858 / 5$ & 3 & 154 & 10800 & 10152 & 62,26 & 210000 & 2015 & 223683,87 & 208026,00 & 1350,82 & 4,880 & G - B & Kş. & PYA - 5 \\
\hline$S, 17$ & $2190 / 1$ & 6 & 168 & 10560 & 9926,4 & 59,09 & 220000 & 2016 & & & 1217,86 & 4,852 & K - B & $\mathrm{Kşs.}$ & PYA - 10 \\
\hline $\mathrm{S}, 17$ & $2190 / 5$ & 4 & 168 & 10800 & 10152 & 60,43 & 229000 & 2016 & 229000 & 212970 & 1267,68 & 4,767 & G - B & Kş. & $S M-200$ \\
\hline $\mathrm{A}, 2$ & $\mathrm{D}, 1885 / 5$ & 2 & 140 & 9240 & 8685,6 & 62,04 & 184000 & 2015 & 195989,67 & 182270,40 & 1301,93 & 4,765 & $G-D$ & Kş. & $\mathrm{M}-20$ \\
\hline $\mathrm{A}, 8$ & $1858 / 1$ & 4 & 162 & 11520 & 10828,8 & 66,84 & 203000 & 2014 & 231805,92 & 215579,51 & 1330,74 & 5,023 & K - B & Kş. & $S M-250$ \\
\hline AVR. & & & 163 & 10087 & 9482 & 58,2 & 187529 & & 206069 & 191644 & 1125,71 & 4,945 & & & \\
\hline
\end{tabular}

Table 5 Intermediate Floor - Intermediate Apartments

\begin{tabular}{|c|c|c|c|c|c|c|c|c|c|c|c|c|c|c|c|}
\hline 芦 & 귤류 & 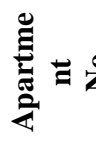 & $\gtrless_{\left(\mathbf{m}^{2}\right)}^{\mathbf{F}}$ & $D_{\text {kyll }}$ & D knet & $D_{\text {kbr }}$ & $\mathbf{D}_{\mathrm{s}}$ & $\mathbf{D}_{\text {syll }}$ & $\mathbf{D}_{\text {sgd }}$ & D snet & $\mathbf{D}_{\text {sbr }}$ & k $(\%)$ & CY & $\mathbf{A K}$ & $\begin{array}{l}\mathbf{U}_{\mathbf{c}} \\
(\mathbf{m})\end{array}$ \\
\hline$S, 1$ & $1591 / 1$ & 3 & 152 & 9900 & 9306 & 61,22 & 172000 & 2014 & 196406,99 & 182658,50 & 1201,70 & 5,051 & K - D & Ar. & SM - 245 \\
\hline $\mathrm{S}, 1$ & $1591 / 3$ & 4 & 152 & 10200 & 9588 & 63,08 & 186000 & 2014 & 212393,60 & 197526,05 & 1299,51 & 4,854 & $G-D$ & Ar. & $S M-270$ \\
\hline$S, 1$ & $2190 / 2$ & 3 & 152 & 9840 & 9249,6 & 60,85 & 160000 & 2013 & 199446,22 & 185484,99 & 1220,30 & 4,987 & $G-B$ & Ar. & $S M-210$ \\
\hline $\mathrm{S}, 2$ & $2146 / 4$ & 3 & 175 & 9900 & 9306 & 53,18 & 176000 & 2014 & 200974,59 & 186906,37 & 1068,04 & ,979 & $G-B$ & Ar. & ÇOP -70 \\
\hline $\mathrm{S}, 2$ & $2146 / 5$ & 4 & 175 & 10800 & 10152 & 58,01 & 205000 & 2015 & 218358,06 & 203072,99 & 1160,42 & 4,999 & G - D & Ar. & ÇOP -25 \\
\hline $\mathrm{S}, 2$ & $2146 / 6$ & 3 & 175 & 9900 & 9306 & 53,18 & 184000 & 2015 & 195989,67 & 182270,40 & 1041,54 & 5,106 & $G-B$ & Ar. & ÇOP -20 \\
\hline $\mathrm{S}, 3$ & $2145 / 3$ & 3 & 181 & 9900 & 9306 & 53,18 & 156000 & 2012 & 2105 & 1958 & 1082,06 & 4,751 & $G-B$ & Ar. & $O-60$ \\
\hline $\mathrm{S}, 3$ & $2145 / 5$ & 3 & 181 & 10500 & 9870 & 54,53 & 180000 & 2013 & 224377,00 & 208670,61 & 1152,88 & 4,730 & $G-B$ & Ar. & ÇOP -30 \\
\hline $\mathrm{S}, 4$ & $1840 / 2$ & 3 & 161 & 9600 & 9024 & 56,05 & 185000 & 20 & 1850 & 17205 & 1068,63 & 5,245 & $\mathrm{~K}-\mathrm{D}$ & Ar. & $\mathrm{O}-325$ \\
\hline $\mathrm{S}, 5$ & $1858 / 3$ & 4 & 172 & 7920 & 7444,8 & 43,28 & 152000 & 2015 & 161904,51 & 150571,20 & 87541 & 4,944 & G - D & Ar. & $\mathrm{SM}-320$ \\
\hline$S, 5$ & $2016 / 3$ & 3 & 172 & 7800 & 7332 & 42,63 & 130000 & 2013 & 1620 & 1507 & 876,20 & 4,865 & $B-K$ & Ar. & $\mathrm{O}-300$ \\
\hline $\mathrm{S}, 5$ & $2016 / 7$ & 4 & 172 & 8760 & 8234,4 & 47,87 & $155 c$ & 2014 & 176 & 1646 & & 5,002 & $G-D$ & Ar. & PYA - 3 \\
\hline$S, 5$ & $2016 / 10$ & 3 & 172 & 7800 & 7332 & 42,63 & 114000 & 2012 & 153896,66 & 143123,89 & 832,12 & 5,123 & $D-G$ & Ar. & $\mathrm{SM}-340$ \\
\hline S, 6 & $2147 / 7$ & 3 & 155 & 9300 & 8742 & 56,40 & 190000 & 2016 & 190000 & 176700 & 1140 & 4,947 & $B-K$ & Ar. & ÇOP - 150 \\
\hline $\mathrm{S}, 6$ & $2147 / 8$ & 3 & 155 & 9240 & 8685,6 & 56,04 & 168000 & 2014 & 191839,38 & 178410,63 & 1151,04 & 4,868 & $\mathrm{~B}-\mathrm{K}$ & Ar. & ÇOP -120 \\
\hline $\mathrm{S}, 7$ & $2147 / 4$ & 4 & 168 & 10200 & 9588 & 57,07 & 203000 & 2016 & 203000 & 188790 & 1123,75 & 5,079 & $\mathrm{D}-\mathrm{K}$ & Ar. & ÇOP -30 \\
\hline $\mathrm{S}, 8$ & $2087 / 6$ & 4 & 162 & 9600 & 9024 & 55,70 & 192000 & 2016 & 192000 & 178560 & 1102,22 & 5,054 & $B-G$ & Ar. & $\mathrm{O}-280$ \\
\hline S,9 & $2021 / 10$ & 3 & 200 & 9600 & 9024 & 45,12 & 192000 & & & & & 5,054 & $G-B$ & Ar. & ÇOP -15 \\
\hline $\mathrm{S}, 12$ & $2022 / 1$ & 4 & 149 & 9000 & 8460 & 56,78 & 175000 & 2015 & 186403,22 & 173355,00 & 1163,46 & 4,880 & G - D & Ar. & $\mathrm{C}-20$ \\
\hline$S, 12$ & $2022 / 2$ & 4 & 149 & 8700 & 8178 & 54,89 & 160000 & 2014 & 182704,18 & 169914,88 & 1140,37 & 4,813 & G - D & Ar. & $C-60$ \\
\hline $\mathrm{S}, 12$ & $2022 / 4$ & 3 & 149 & 8880 & 8347,2 & 56,02 & & 2015 & 186403,22 & 173355,00 & 1163,46 & & $G-B$ & Ar. & $\mathrm{C}-110$ \\
\hline $\mathrm{S}, 13$ & $2086 / 3$ & 3 & 124 & 7800 & 7332 & 59,13 & 125000 & 2013 & 155817,36 & 144910,15 & 1168,63 & 5,060 & $G-B$ & Ar. & ÇOP -110 \\
\hline
\end{tabular}




\begin{tabular}{|c|c|c|c|c|c|c|c|c|c|c|c|c|c|c|c|}
\hline$S, 14$ & $\mathrm{D}, 105 / 6$ & 4 & 160 & 9720 & 9136,8 & 57,10 & 145000 & 2012 & 195745,75 & 182043,55 & 1137,77 & 5,019 & G - D & Ar. & SM - 130 \\
\hline$S, 16$ & $1587 / 4$ & 3 & 100 & 5100 & 4794 & 47,94 & 88000 & 2016 & 88000 & 81840 & 818,4 & 5,858 & $B-K$ & Ar. & $O-150$ \\
\hline $\mathrm{A}, 3$ & $\mathrm{D}, 1236 / 1$ & 3 & 140 & 8400 & 7896 & 56,4 & 165000 & 2015 & 175751,61 & 163449,00 & 1167,49 & 4,831 & $\mathrm{~K}-\mathrm{D}$ & Ar. & $M-15$ \\
\hline A,5 & $1580 / 1$ & 3 & 136 & 8100 & 7614 & 55,98 & 153000 & 2015 & 162969,67 & 151561,80 & 1114,42 & 5,024 & $\mathrm{~K}-\mathrm{D}$ & Ar. & SM -150 \\
\hline$A, 6$ & $2085 / 6$ & 3 & 135 & 7800 & 7332 & 54,31 & 150000 & 2015 & 159774,19 & 148590,00 & 1100,67 & 4,934 & $B-K$ & Ar. & $\mathrm{O}-120$ \\
\hline VR. & & & 158,3 & 046,67 & 8503,87 & 54,02 & 164296,30 & & 183733,19 & 170871,86 & 6852,83 & 4,995 & & & \\
\hline
\end{tabular}

Table 6 Top Floor - Intermediate Apartments

\begin{tabular}{|c|c|c|c|c|c|c|c|c|c|c|c|c|c|c|c|}
\hline : & 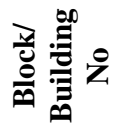 & 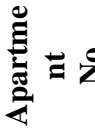 & $\underset{\left(\mathbf{m}^{2}\right)}{\mathbf{F}}$ & $D_{\text {kyl }}$ & D $_{\text {knet }}$ & $D_{\text {kbr }}$ & $\mathbf{D}_{\mathrm{s}}$ & $\mathbf{D}_{\text {syll }}$ & $\mathbf{D}_{\text {sgd }}$ & $\mathbf{D}_{\text {snet }}$ & $\mathbf{D}_{\text {sbr }}$ & k (\%) & CY & AK & $\begin{array}{l}\mathbf{U}_{\mathbf{c}} \\
(\mathbf{m})\end{array}$ \\
\hline $\mathrm{S}, 2$ & $2146 / 4$ & 5 & 175 & 9780 & 9193,2 & 52,53 & 190000 & 2016 & 190000 & 176700 & 1009,71 & 5,203 & $G-B$ & Ar. & ÇOP -70 \\
\hline $\mathrm{S}, 3$ & $2145 / 4$ & 5 & 181 & 10440 & 9813,6 & 54,22 & 215000 & 2016 & 215000 & 199950 & 1104,70 & 4,908 & $G-B$ & Ar. & ÇOP -25 \\
\hline $\mathrm{S}, 3$ & $2145 / 5$ & 6 & 181 & 10200 & 9588 & 52,97 & 168000 & 2013 & 209418,53 & 194759,24 & 1076,02 & 4,923 & $G-D$ & Ar. & ÇOP - 30 \\
\hline $\mathrm{S}, 5$ & $2016 / 3$ & 5 & 172 & 7500 & 7050 & 40,99 & 148000 & 2015 & 157643,87 & 146608,80 & 852,38 & 4,809 & B $-\mathrm{K}$ & Ar. & $\mathrm{O}-300$ \\
\hline $\mathrm{S}, 6$ & $2147 / 8$ & 5 & 155 & 9120 & 8572,8 & 55,31 & 187000 & 2016 & 187000 & 173910 & 1122 & 4,929 & B $-\mathrm{K}$ & Ar. & ÇOP -120 \\
\hline $\mathrm{S}, 8$ & $2087 / 2$ & 5 & 162 & 9600 & 9024 & 55,70 & 153000 & 2013 & 190720,45 & 177370,02 & 1094,88 & 5,088 & $K-D$ & Ar. & $\mathrm{O}-240$ \\
\hline $\mathrm{S}, 9$ & $2021 / 5$ & 5 & 200 & 9780 & 9193,2 & 45,97 & 167000 & 2014 & 190697,48 & 177348,66 & 886,74 & 5,184 & $G-B$ & Ar. & $C-335$ \\
\hline $\mathrm{S}, 9$ & $2021 / 7$ & 5 & 200 & 11040 & 10377,6 & 51,89 & 216000 & 2016 & 216000 & 200880 & 1004,40 & 5,166 & $G-B$ & Ar. & PYA - 10 \\
\hline $\mathrm{S}, 9$ & $2021 / 8$ & 6 & 200 & 11160 & 10490,4 & 52,45 & 214000 & 2015 & 227944,51 & 211988,39 & 1059,94 & 4,949 & $G-D$ & Ar. & PYA - 10 \\
\hline $\mathrm{S}, 12$ & $2022 / 4$ & 6 & 149 & 8520 & 8008,8 & 53,75 & 154000 & 2014 & 175852,77 & 163543,08 & 1097,60 & 4,897 & $G-D$ & Ar. & $C-110$ \\
\hline$S, 13$ & $2086 / 1$ & 6 & 124 & 7500 & 7050 & 56,85 & 146000 & 2015 & 155513,54 & 144627,60 & 1166,35 & 4,875 & $G-D$ & Ar. & ÇOP -115 \\
\hline$S, 14$ & $\mathrm{D}, 105 / 1$ & 6 & 160 & 9600 & 9024 & 56,4 & 140000 & 2012 & 188995,90 & 175766,18 & 1098,54 & 5,134 & $G-D$ & Ar. & $\mathrm{SM}-140$ \\
\hline$S, 15$ & $1858 / 7$ & 6 & 154 & 8700 & 8178 & 53,10 & 165000 & 2015 & 175751,61 & 163449,00 & 1061,36 & 5,003 & $G-D$ & Ar. & $S M-260$ \\
\hline$S, 16$ & $1586 / 8$ & 5 & 100 & 4680 & 4399,2 & 43,99 & 82000 & 2016 & 82000 & 76260 & 762,6 & 5,769 & $D-G$ & Ar. & PYA - 20 \\
\hline AVR. & & & 165 & 9116 & 8568,77 & 51,87 & 167500 & & 183038,48 & 170225,78 & 1028,37 & 5,060 & & & \\
\hline
\end{tabular}

Table 7 Top Floor - Corner Apartments

\begin{tabular}{|c|c|c|c|c|c|c|c|c|c|c|c|c|c|c|c|}
\hline 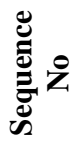 & 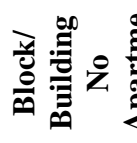 & 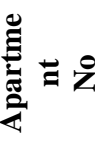 & $\begin{array}{c}\mathbf{F} \\
\left(\mathbf{m}^{2}\right)\end{array}$ & $D_{\text {kyll }}$ & D $_{\text {knet }}$ & $D_{k b r}$ & $\mathbf{D}_{\mathrm{s}}$ & $\mathbf{D}_{\text {syll }}$ & $\mathbf{D}_{\text {sgd }}$ & D $_{\text {snet }}$ & $\mathbf{D}_{\text {sbr }}$ & k $(\%)$ & CY & AK & $\begin{array}{l}\mathbf{U}_{\mathbf{c}} \\
(\mathbf{m})\end{array}$ \\
\hline $\mathrm{S}, 1$ & $1591 / 1$ & 6 & 152 & 9900 & 9306 & 61,22 & 183000 & 2015 & 194924,51 & 181279,80 & 1192,63 & 5,133 & $\mathrm{~K}-\mathrm{B}$ & Kş. & $\mathrm{SM}-245$ \\
\hline $\mathrm{S}, 1$ & $1591 / 3$ & 5 & 152 & 10200 & 9588 & 63,08 & 184000 & 2014 & 210109,80 & 195402,12 & 1285,54 & 4,907 & $G-B$ & Kş. & $\mathrm{SM}-270$ \\
\hline $\mathrm{S}, 1$ & $2190 / 4$ & 6 & 152 & 10320 & 9700,8 & 63,82 & 182000 & 2014 & 207826,00 & 193278,18 & 1271,57 & 5,019 & $G-D$ & Kş. & $\mathrm{SM}-260$ \\
\hline $\mathrm{S}, 5$ & $2016 / 8$ & 5 & 172 & 8700 & 8178 & 47,55 & 185000 & 2016 & 185000 & 172050 & 1000,29 & 4,753 & $G-B$ & Kş. & PYA - 3 \\
\hline $\mathrm{S}, 6$ & $2145 / 6$ & 5 & 155 & 9420 & 8854,8 & 57,13 & 170000 & 2014 & 194123,19 & 180534,56 & 1164,74 & 4,905 & $G-B$ & Kş. & ÇOP -55 \\
\hline $\mathrm{S}, 17$ & $2190 / 1$ & 9 & 168 & 10500 & 9870 & 58,75 & 225000 & 2016 & 225000 & 209250 & 1245,54 & 4,717 & $\mathrm{~K}-\mathrm{B}$ & Kş. & PYA -10 \\
\hline $\mathrm{A}, 1$ & $1584 / 7$ & 11 & 136 & 7800 & 7332 & 53,91 & 122000 & 2013 & 152077,74 & 141432,30 & 1039,94 & 5,184 & $\mathrm{~K}-\mathrm{D}$ & Kş. & $\mathrm{O}-25$ \\
\hline $\mathrm{A}, 5$ & $1580 / 1$ & 6 & 136 & 7920 & 7444,8 & 54,74 & 150000 & 2015 & 159774,19 & 148590,00 & 1092,57 & 5,010 & $\mathrm{~K}-\mathrm{B}$ & Kş. & $\mathrm{SM}-150$ \\
\hline AVR. & & & 153 & 9345 & 8784,30 & 57,53 & 175125 & & 191104,43 & 177727,12 & 1161,60 & 4,954 & & & \\
\hline
\end{tabular}

In this way, integrated capitalisation ratio of flats in seperate locations is calculated.

$$
\begin{aligned}
& D_{\text {knet }}=D_{\text {kyl }}-Z G_{\text {kira }} \\
& D_{\text {snet }}=D_{\text {sgd }}-Z G_{\text {satts }} \\
& D_{\text {sgd }}=D_{s}\left(\frac{T F E R}{T F E R_{s}}\right) \\
& D_{k b r}=\frac{D_{\text {knet }}}{F}
\end{aligned}
$$

$$
\begin{aligned}
& D_{\text {sbr }}=\frac{D_{\text {snet }}}{F} \\
& k=\frac{D_{\text {knet }}}{D_{\text {snet }}}
\end{aligned}
$$

\section{CONCLUSION}

Similar residences' integrated capitalisation ratios located in project field calculated as in Table 8, utilizing correlations from 5 to 10 
Table 8 Residences Arranged According to Their Locations (with respect to unit rental andselling values)

\begin{tabular}{|c|c|c|c|c|c|c|c|c|c|c|c|}
\hline Location & $\underset{\left(\mathbf{m}^{2}\right)}{\mathbf{F}}$ & Dkyl & D $_{\text {knet }}$ & D $_{\text {kbr }}$ & $\mathbf{D}_{\mathrm{s}}$ & $D_{\text {sgd }}$ & D $_{\text {snet }}$ & $\mathbf{D}_{\text {sbr }}$ & $\mathbf{k}(\%)$ & $\begin{array}{l}\text { Index } \\
\text { Rent }\end{array}$ & $\begin{array}{c}\text { Index } \\
\text { Sale }\end{array}$ \\
\hline Ground-Inte & 157 & 8264,12 & 7768,27 & 50,23 & 140559 & 168250,77 & 156473,22 & 996,64 & 4,989 & 100,00 & 100,00 \\
\hline Top Flat-Intermediate & 165 & 9116 & 8568,77 & 51,87 & 167500 & 183038,48 & 170225,78 & 1031,67 & 5,060 & 103,26 & 103,51 \\
\hline $\begin{array}{l}\text { Intermediate Flat- } \\
\text { Intermediate }\end{array}$ & 158 & 9046,67 & 8503,87 & 54,02 & 164296,30 & 183733,19 & 170871,86 & 1081,47 & 4,995 & 105,55 & 108,51 \\
\hline Ground-Corner & 145 & 8668 & 8148 & 56,28 & 157333,3 & 174756,23 & 162521,29 & 1120,84 & 5,073 & 112,04 & 112,46 \\
\hline Top Flat-Corner & 153 & 9345 & 8784,30 & 57,53 & 175125 & 191104,43 & 177727,12 & 1161,60 & 4,954 & 114,53 & 116,55 \\
\hline Intermediate Flat-Corner & 163 & 10087 & 9482 & 58,2 & 187529 & 206069 & 191644 & 1175,71 & 4,945 & 115,87 & 117,97 \\
\hline
\end{tabular}

Residences shown in table with respect to their locations are arranged according to Unit Sale (Dsbr) and Unit Rental (Dkbr) Values. When examining rental and selling indexes in last two columns of Table 8 , following conclusions can be reached;

a) Unit Sale Valuesare always more than Unit Rental Values.

b) Because of first clause, increase rate of sale value is greater than increase rate of rental value.

c) In locational sequencing, residences located in the corner are more valuable than residences located in intermediate floors.

d) Thereare no considerable diferences among integrated capitalisation ratios.

e) Most valuable place among corner and intermediate located real properties is intermediate floor.

f) Value-based locational sequencing is Ground Floor Intermediate - Top Floor Intermediate Intermediate Floor Intermediate-Ground Floor Corner - Top Floor Corner - Intermediate Floor Corner respectively.

g) Value of intermediate floor corner residence is about $18 \%$ more valuable than ground floor intermediate residance.

The $\mathrm{k}$ rates by the calculations can be used in similar characteristics areas.

By utilizing similar residences' determined integrated capitalisation ratio, sale values of residences located in project field whose renting revenues are knownand also renting values of residences whose sale prices are known can be determined. In order to calculate sale values

$D_{\text {satts }}=\frac{G_{n e t}}{k_{\text {büt }}}$

Correlation can be applied for determinig flats' sale values by utilizing flats' renting revenues.

\section{REFERENCES}

Açlar \& Çağdaş, 2002. Taşınmaz (Gayrimenkul) Değerlemesi, Birinci Baskı TMMOB Harita ve Kadastro Mühendisleri Odası, Ankara,

Çağdaş, Ders Notları, Yıldız Teknik ÜniversitesiDavutpaşa Kampüsü, Slayt Notlar,

Çelik, Ders Notları, Gümüşhane ÜniversitesiGümüşhane Kampüsü, Slayt Notlar,

Demir, Ders Notları, Yıldız Teknik ÜniversitesiDavutpaşa Kampüsü, Slayt Notlar,

Erol Köktürk \& Erdal Köktürk, 2011. Taşınmaz Değerlemesi, Birinci Bask1, stanbul,

Ertaş, Ders Notları, 2015. Selçuk Üniversitesi-Alaeddin Keykubat Yerleşkesi Selçuklu/Konya, Slayt Notlar,

Kuster J. 1998. Factors Determining Housing Prices in Zurich, s:163-179, VR/39/8, Zürih

Tanrıvermiş, 2010. Gayrimenkul Değerleme Esasları, Lisanslama Sınavları Çalışma Kitapları, Ders Kodu: 1014, Ankara Üniversitesi Uygulamalı Bilimler Fakültesi Gayrimenkul Geliştirme ve Yönetimi Bölümü,

UDES, 2016. Uluslararası Değerleme Standartları, Genel Değerleme Kavram ve lkeleri, S.11, 4.4

URL 1. Türkiye statistik Kurumu, http://www,tuik,gov,tr/UstMenu,do?metod= ,Tüketici Fiyat Endeksi $(2003=100$ 


\section{ABBREVIATIONS}

A,1 : Apartment No:1

A,2 : Apartment No:2

A,3 : Apartment No:3

A,4 : Apartment No:4

Copyright (C) International Journal of Engineering and Geosciences (IJEG). All rights reserved, including the making of copies unless permission is obtained from the copyright proprietors.
ZGkira : Compulsory Expenses to Renting (Real Estate Agent's Commission + Collection Difficulties + Real Estate Tax and Expenditures+ Permanent Equipment Expenses) $=\% 6$

ZGsatış : Compulsory Expenses for Sale(Real Estate Agent's Commission + Sale Tax and Expenditures + Income Tax $)=\% 7$
A,5 : Apartment No:5

A,6 : Apartment No:6

A,7 : Apartment No:7

A,8 : Apartment No:8

A/B No: Block/Building Number

AK : Location in Block

Ar. : Intermediate

B : West

kbüt : IntegratedCapitalisation Ratio

C : Mosque

CY : Frontage

ÇOP : Playground

D : East

Dk : Rental Value (TL) / month

Dkbr : Unit Rental Value (TL)

Dknet : Annual Net Rental Value (TL) / year

Dkyıl : Annual Rental Value (TL) / year

D No: Flat Number

Dsyıl : SaleYear (TL)

Ds : Sale Value (TL)

Dsgd : Sale Value Updated according to Consumer

Price Index (TL)

Dsbr : Unit Sale Value (TL)

Dsnet : Net Sale Value (TL)

F : Area $\mathrm{m} 2$

G : South

K : North

Kș. : Corner

k : Rental Capitalisation Ratio (\%)

M : Market

O : School

ORT : Average

PYA : Park

S,1 : Housing Estate Number

S/A : Housing Estate/Apartment

SM : Supermarket

TFER : Consumer Price Index of April 2016

TFERS : Price Index of June of Sale's Year

Uc : Distance to Closest Attraction Point(m) 